\title{
Short range correlations in relativistic nuclear matter models
}

\author{
P.K. Panda, João da Providência and Constança Providência \\ Departamento de Física, Universidade de Coimbra, 3000 Coimbra, Portugal
}

(Dated: July 19, 2018)

\begin{abstract}
Short range correlations are introduced using unitary correlation method in a relativistic approach to the equation of state of the infinite nuclear matter in the framework of the Hartree-Fock approximation. It is shown that the correlations give rise to an extra node in the ground-state wave-function in the nucleons, contrary to what happens in non-relativistic calculations with a hard core. The effect of the correlations in the ground state properties of the nuclear matter and neutron matter is studied. The nucleon effective mass and equation of state (EOS) are very sensitive to short range correlations. In particular, if the pion contact term is neglected a softening of the EOS is predicted. Correlations have also an important effect on the neutron matter EOS which presents no binding but only a very shallow minimum contrary to the Walecka model.
\end{abstract}

We discuss the role of short range correlations in a relativistic approach to the description of nuclear matter. Several procedures may be used to introduce short range correlations into the model wave function. All have something in common with the so called $e^{S}$ method or coupled-cluster expansion, introduced by Coester and Kümmel [1] and most elegantly and extensively developed and applied to diverse quantal systems by R. Bishop [2].

In this short note, we consider the unitary operator method as proposed by Villars [3], which automatically guarantees that the correlated state is normalized. The general idea of introducing short range correlations in systems with short range interactions exists for a long time [4, 5] but has not been pursued for the relativistic case.

Non-relativistic calculations based on realistic NN potentials predict equilibrium points which do not reproduce simultaneously the binding energy and saturation density. Either the saturation density is reproduced but the binding energy is too small, or the binding energy is reproduced at too high a density [6]. In order to solve this problem, the existence of a repulsive potential or density-dependent repulsive mechanism [7] is usually assumed. Due to Lorentz covariance and self-consistency, relativistic mean field theories [8] include automatically contributions which are equivalent to $n$-body repulsive potentials in non-relativistic approaches. The relativistic quenching of the scalar field provides a mechanism for saturation, though, by itself it may lead to too small an effective mass and too large incompressibility of nuclear matter, a situation which is encountered in the Walecka model $[8]$.

In the non-relativistic case, we find a wound in the relative wave function if the vector interaction is stronger than the scalar interaction. However, this may not be the case if the interaction is strong enough for short distances. Then, the effective potential can actually turn attractive at short distances and the wave function may well have a node, as proposed by V. Neudatchin [9] and advocated by S. Moszkowski [10], on the basis of the the quark structure of the nucleon. The situation with short range correlations may be more subtle than might be thought from a simple nonrelativistic model. We show that a short range node in the relative wave function may be encountered in relativistic models, although it remains to be seen to what extent the relativistic description simulates the quark structure.

In non-relativistic models the saturation arises from the interplay between a long range attraction and a short range repulsion, so strong that it is indispensable to take short range correlations into account. In relativistic mean field models, the parameters are phenomenologically fitted to the saturation properties of nuclear matter. Although in this approach short range correlation effects may be accounted for, to some extent, by the model parameters, it is our aim to study explicitly the consequences of actual short range correlations. In a previous publication [12] we have discussed the effect of the correlations in the ground state properties of nuclear matter in the framework of the Hartree-Fock approximation using an effective Hamiltonian derived from the $\sigma-\omega$ Walecka model. We have shown, for interactions mediated only by sigma and omega mesons, that the equation of state (EOS) becomes considerably softer when correlations are taken into account, provided the correlation function is treated variationally, always paying careful attention to the constraint imposed by the "healing distance" requirement. In the present note we will work within the same approach and will include also the exchange of pions and $\rho$-mesons. Preliminary results of the present work have been presented in 11]

We start by considering the effective Hamiltonian 12

$$
H=\int \psi_{\alpha}^{\dagger}(\vec{x})(-i \vec{\alpha} \cdot \vec{\nabla}+\beta M)_{\alpha \beta} \psi_{\beta}(\vec{x}) d \vec{x}+\frac{1}{2} \int \psi_{\alpha}^{\dagger}(\vec{x}) \psi_{\gamma}^{\dagger}(\vec{y}) V_{\alpha \beta, \gamma \delta}(|\vec{x}-\vec{y}|) \psi_{\delta}(\vec{y}) \psi_{\beta}(\vec{x}) d \vec{x} d \vec{y},
$$

where the exchange of $\sigma, \omega, \rho$ and $\pi$ mesons is taken into account, so that

$$
V_{\alpha \beta, \gamma \delta}(r)=\sum_{i=\sigma, \omega, \rho, \pi} V_{\alpha \beta, \gamma \delta}^{i}(r)
$$


with

$$
\begin{gathered}
V_{\alpha \beta, \gamma \delta}^{\sigma}(r)=-\frac{g_{\sigma}^{2}}{4 \pi}(\beta)_{\alpha \beta}(\beta)_{\gamma \delta} \frac{e^{-m_{\sigma} r}}{r} \\
V_{\alpha \beta, \gamma \delta}^{\omega}(r)=\frac{g_{\omega}^{2}}{4 \pi}\left(\delta_{\alpha \beta} \delta_{\gamma \delta}-\vec{\alpha}_{\alpha \beta} \cdot \vec{\alpha}_{\gamma \delta}\right) \frac{e^{-m_{\omega} r}}{r} \\
V_{\alpha \beta, \gamma \delta}^{\rho}(r)=\frac{g_{\rho}^{2}}{4 \pi}\left(\delta_{\alpha \beta} \delta_{\gamma \delta}-\vec{\alpha}_{\alpha \beta} \cdot \vec{\alpha}_{\gamma \delta}\right) \vec{\tau}_{1} \cdot \vec{\tau}_{2} \frac{e^{-m_{\rho} r}}{r} .
\end{gathered}
$$

An interaction of the form 13

$$
V_{\alpha \beta, \gamma \delta}^{\pi}(\vec{r})=\frac{1}{3}\left[\frac{f_{\pi}}{m_{\pi}}\right]^{2}\left(\Sigma_{i}\right)_{\alpha \beta}\left(\Sigma_{i}\right)_{\gamma \delta} \vec{\tau}_{1} \cdot \vec{\tau}_{2}\left[\frac{4 \pi}{m_{\pi}^{3}} \delta(\vec{r})-\frac{e^{-m_{\pi} r}}{m_{\pi} r}\right],
$$

where $\Sigma_{i}=\alpha_{i} \gamma_{5}$, describes the exchange of $\pi$ mesons. The first term in the above is the repulsive contact interaction and the second term is an attractive Yukawa potential. This can be rewritten in momentum space as

$$
V_{\alpha \beta \gamma \delta}^{\pi}(\vec{q})=\frac{1}{3}\left[\frac{f_{\pi}}{m_{\pi}}\right]^{2}\left(\Sigma_{i}\right)_{\alpha \beta}\left(\Sigma_{i}\right)_{\gamma \delta} \vec{\tau}_{1} \cdot \vec{\tau}_{2}\left[\frac{q^{2}}{q^{2}+m_{\pi}^{2}}\right] .
$$

In equation (11), $\psi$ is the nucleon field interacting through the scalar and vector potentials and $\vec{\alpha}, \beta$ are the Diracmatrices. The equal time quantization condition for the nucleons reads, $\left[\psi_{\alpha}(\vec{x}, t), \psi_{\beta}(\vec{y}, t)^{\dagger}\right]_{+}=\delta_{\alpha \beta} \delta(\vec{x}-\vec{y})$, where the indices $\alpha$ and $\beta$ refer to the spin. The field expansion for the field $\psi$ at time $\mathrm{t}=0$ reads 14 ]

$$
\psi(\vec{x})=\frac{1}{\sqrt{V}} \sum_{r, k}\left[U_{r}(\vec{k}) c_{r, \vec{k}}+V_{r}(-\vec{k}) \tilde{c}_{r,-\vec{k}}^{\dagger}\right] e^{i \vec{k} \cdot \vec{x}}
$$

where $U_{r}$ and $V_{r}$ are

$$
U_{r}(\vec{k})=\left(\begin{array}{c}
\cos \frac{\chi(\vec{k})}{2} \\
\vec{\sigma} \cdot \hat{k} \sin \frac{\chi(\vec{k})}{2}
\end{array}\right) u_{r} ; \quad V_{r}(-\vec{k})=\left(\begin{array}{c}
-\vec{\sigma} \cdot \hat{k} \sin \frac{\chi(\vec{k})}{2} \\
\cos \frac{\chi(\vec{k})}{2}
\end{array}\right) v_{r} .
$$

For free spinor fields, we have $\cos \chi(\vec{k})=M / \epsilon(\vec{k}), \sin \chi(\vec{k})=|\vec{k}| / \epsilon(\vec{k})$ with $\epsilon(\vec{k})=\sqrt{\vec{k}^{2}+M^{2}}$. However, we will deal with interacting fields so that we take the ansatz $\cos \chi(\vec{k})=M^{*}(\vec{k}) / \epsilon^{*}(\vec{k}), \sin \chi(\vec{k})=\left|\vec{k}^{*}\right| / \epsilon^{*}(\vec{k})$, with $\epsilon^{*}(\vec{k})=$ $\sqrt{{\overrightarrow{k^{*}}}^{2}+M^{*}(\vec{k})}$, where $\vec{k}^{*}$ and $M^{*}(\vec{k})$ are the effective momentum and the effective mass, respectively, determined self-consistently by the Hartree-Fock (HF) prescription. The vacuum $|0\rangle$ is defined through $c_{r, \vec{k}}|0\rangle=\tilde{c}_{r, \vec{k}}^{\dagger}|0\rangle=0$; one-particle states are written $|\vec{k}, r\rangle=c_{r, \vec{k}}^{\dagger}|0\rangle$; two-particle and three-particle uncorrelated states are written, respectively as $\left|\vec{k}, r ; \vec{k}^{\prime}, r^{\prime}\right\rangle=c_{r, \vec{k}}^{\dagger} c_{r^{\prime}, \vec{k}^{\prime}}^{\dagger}|0\rangle$, and $\left|\vec{k}, r ; \vec{k}^{\prime}, r^{\prime} ; \vec{k}^{\prime \prime}, r^{\prime \prime}\right\rangle=c_{r, \vec{k}}^{\dagger} c_{r^{\prime}, \vec{k}^{\prime}}^{\dagger} c_{r^{\prime \prime}, \vec{k}^{\prime \prime}}^{\dagger}|0\rangle$, and so on.

We now introduce the short range correlations through the unitary operator method. The correlated wave function [15] is $|\Psi\rangle=e^{i \Omega}|\Phi\rangle$ where $|\Phi\rangle$ is a Slater determinant and $\Omega$ is, in general, a $n$-body Hermitian operator, splitting into a 2-body part, a 3-body part, etc.. The expectation value of $H$ is

$$
E=\frac{\langle\Psi|H| \Psi\rangle}{\langle\Psi \mid \Psi\rangle}=\frac{\left\langle\Phi\left|e^{-i \Omega} H e^{i \Omega}\right| \Phi\right\rangle}{\langle\Phi \mid \Phi\rangle}
$$

In the present calculation, we only take into account two-body correlations. Let us denote the two-body correlated wave function by $\left|\overrightarrow{k, r} r \vec{k}^{\prime}, r^{\prime}\right\rangle=e^{i \Omega}\left|\vec{k}, r ; \vec{k}^{\prime}, r^{\prime}\right\rangle \approx f_{12}\left|\vec{k}, r ; \vec{k}^{\prime}, r^{\prime}\right\rangle$ where $f_{12}$ is the short range correlation factor, the so-called Jastrow factor [16]. For simplicity, we consider $f_{12}=f\left(\vec{r}_{12}\right), \vec{r}_{12}=\vec{r}_{1}-\vec{r}_{2}$, and $f(r)=1-(\alpha+\beta r) e^{-\gamma r}$ where $\alpha, \beta$ and $\gamma$ are parameters. The choice of a real function for the unitary operator has to be supplemented by a normalization condition (see eq. (8]) which assures unitarity to leading cluster order.

The important effect of the short range correlations is the expression for the correlated ground-state energy. Here, in the leading order of the cluster expansion, the interaction matrix element $\left\langle\vec{k}, r ; \vec{k}^{\prime}, r^{\prime}\left|V_{12}\right| \vec{k}, r ; \vec{k}^{\prime}, r^{\prime}\right\rangle$ of the HF expression is replaced by $\left\langle\overrightarrow{\vec{k}, r ; \vec{k}^{\prime}, r^{\prime}}\left|V_{12}+t_{1}+t_{2}\right| \overrightarrow{\vec{k}, r ; \vec{k}^{\prime}, r^{\prime}}\right\rangle-\left\langle\vec{k}, r ; \vec{k}^{\prime}, r^{\prime}\left|t_{1}+t_{2}\right| \vec{k}, r ; \vec{k}^{\prime}, r^{\prime}\right\rangle$, where $t_{i}$ is the kinetic 
energy operator of particle $i$. As argued by Moszkowski [17] and Bethe [18], it is expected that the true ground-state wave function of the nucleus, containing correlations, coincides with the independent particle, or HF wave function, for inter particle distances $r \geq r_{h}$, where $r_{h} \approx 1 \mathrm{fm}$ is the so-called "healing distance". This behavior is a consequence of the restrictions imposed by the Pauli Principle. A natural consequence of having the correlations introduced by a unitary operator is a normalization constraint on $f(r)$,

$$
\int\left(f^{2}(r)-1\right) d^{3} r=0 .
$$

The correlated ground state energy of symmetric nuclear matter reads

$$
\begin{aligned}
\mathcal{E} & =\frac{\nu}{\pi^{2}} \int_{0}^{k_{F}} k^{2} d k[|k| \sin \chi(k)+M \cos \chi(k)]+\frac{\tilde{F}_{\sigma}(0)}{2} \rho_{s}^{2}+\frac{\tilde{F}_{\omega}(0)}{2} \rho_{B}^{2} \\
& -\frac{4}{(2 \pi)^{4}} \int_{0}^{k_{f}} k^{2} d k k^{\prime 2} d k^{\prime}\left\{[|k| \sin \chi(k)+2 M \cos \chi(k)] I\left(k, k^{\prime}\right)+|k| \sin \chi\left(k^{\prime}\right) J\left(k, k^{\prime}\right)\right\} \\
& +\frac{1}{(2 \pi)^{4}} \int_{0}^{k_{f}} k d k k^{\prime} d k^{\prime}\left[\sum_{i=\sigma, \omega, \rho, \pi} A_{i}\left(k, k^{\prime}\right)+\cos \chi(k) \cos \chi\left(k^{\prime}\right) \sum_{i=\sigma, \omega, \rho, \pi} B_{i}\left(k, k^{\prime}\right)\right. \\
& \left.+\sin \chi(k) \sin \chi\left(k^{\prime}\right) \sum_{i=\sigma, \omega, \rho, \pi} C_{i}\left(k, k^{\prime}\right)\right]
\end{aligned}
$$

where $A_{i}, B_{i}, C_{i}, I$ and $J$ are exchange integrals defined in the appendix. In the above equation, the first term comes from the kinetic contribution, the second and third terms come respectively from the $\sigma$ and $\omega$ direct contributions to the correlated potential energy, the other terms arise from the exchange correlation contribution to the kinetic energy, and from the meson exchange contributions to the correlated potential energy. The direct term of the correlation contribution to the kinetic energy vanishes due to (8), and $\rho_{B}$ and $\rho_{s}$ are, respectively, the baryon and the scalar densities.

The couplings $g_{\sigma}, g_{\omega}, g_{\rho}, g_{\pi}$, the meson masses, $m_{i}, i=\sigma, \omega, \rho, \pi$ and the three parameters specifying the short range correlation function, $\alpha, \beta$ and $\gamma$ have to be fixed. The couplings $g_{\sigma}$ and $g_{\omega}$ are chosen so as to reproduce the ground state properties of nuclear matter. For the $\rho$ and $\pi$-meson couplings we take the usual values $g_{\rho}^{2} / 4 \pi=0.55$ and $f_{\pi}^{2} / 4 \pi=0.08$. We choose $m_{\sigma}=550 \mathrm{MeV}, m_{\omega}=783 \mathrm{MeV}, m_{\rho}=770 \mathrm{MeV}$ and $m_{\pi}=138 \mathrm{MeV}$. The normalization condition (8) determines $\beta$. We fix $\alpha$ by minimizing the energy. The parameter $\gamma$ is such that it reproduces a reasonable healing distance, assuming that this quantity decreases as $k_{F}$ increases. Therefore, we assume that $\gamma$ depends on $k_{F}$ according to $\gamma=a_{1}+a_{2} k_{F} / k_{F 0}$, where the parameters $a_{1}$ and $a_{2}$ are conveniently chosen.

In tables [ and II] we have tabulated the parameters used in our calculation together with the relative effective mass $M^{*} / M$, the kinetic energy $\mathcal{T} / \rho_{B}-M$, the direct and exchange parts of the potential energy $\left(\mathcal{V}_{d} / \rho_{B}\right.$ and $\mathcal{V}_{e} / \rho_{B}$ respectively) with correlation, and the correlation contribution to the kinetic energy $\mathcal{T}^{C} / \rho_{B}$, all calculated at the saturation point. Notice that a HF calculation produces an EOS which is stiffer than the one obtained at the Hartree level. However, correlations reduce the effective mass and soften the EOS. In fact, the contribution of direct and exchange correlation terms are of the same order of magnitude of the other terms in the energy per particle. Moreover, the values of the couplings $g_{\sigma}$ and $g_{\omega}$ which reproduce the saturation density and binding energy strongly depend on the correlations, being considerably reduced by their presence, which is quite a remarkable fact. Hence, short-range correlations cannot be disregarded.

The correlation function $f(r)$ is plotted in figure 1 as a function of the relative distance for two different situations: in one calculation the contact term coming from the pion contribution was included and the other curve was obtained excluding it. It is clear from both curves that the correlations give rise to an extra node in the dependence of the ground-state wave-function on the relative coordinate, contrary to what generally happens in non-relativistic calculations with a hard core, when the wave function acquires a wound. However, both curves are rather different behaviors: when the contact term is included the node appears very close to zero and the correlation function has a quite flat behavior. This is a sign that the correlation function used was not flexible enough to respond simultaneously to the repulsive contact interaction and to the attractive component of the interaction.

We have computed the binding energies as function of the density for the Hartree, HF and HF + Corr and compared with the quark-meson-coupling model (QMC) [19], as can be seen from fig. 2. The inclusion of correlations make the equation of state (EOS) softer than Hartree or HF calculations, if the contact term of the pion contribution is neglected. We also see that the inclusion of the $\rho$ and $\pi$-mesons brings extra softness to the EOS. However, the curve 
which describes the model with contact term and with the correlations taken into account shows a very stiff behavior. This is due to the fact that a very simplified parametrization of the correlation function was used, which was not flexible enough to respond to the repulsive and to attractive components of the interaction. A softer EOS around nuclear matter saturation density is also provided by QMC.

In Fig. 3, we plot the effective mass versus density of nuclear matter. If correlations are included and the contact term is neglected the effective mass does not decrease so fast with the increase of density as in a Hartree or, even worse, HF calculation. This explains the softer behavior of the EOS with correlations. However, the variation of the effective mass with density is still smaller within the QMC model.

Correlations also affect the behavior of the neutron matter equation of state (EoS), which is plotted in Fig.4. In this calculation we include the four mesons $\sigma, \omega, \rho$ and $\pi$. We perform the calculation with and without short range correlations and with and without the delta term of the $\pi$ contribution. The delta term makes the equation of state very hard in both calculations: with and without short range correlations. We have already discussed that the correlation function should be more flexible to deal with the delta term. Both EoS without the delta term show an unrealistic behavior: either binding in the Hartree Fock calculation or a shallow minimum in the HF plus correlations calculation. Neutron star observation data are compatible with a zero density surface which would not be the case if a minimum at finite density would occur in the neutron star EoS. It is, however important to point out that the inclusion of correlations almost lifts this behavior of the neutron EoS typical of the Walecka model [20].

We stress that a node occurs in the relative wave function, and the EOS becomes softer, when the energy is optimized with respect to variations of $\alpha$. However, if $\alpha$ is set equal to 1 , so that the node in the relative wave function is replaced by a wound, the EOS remains stiff. A variational treatment is therefore essential.

We conclude that the explicit introduction of correlations has important effects. The behavior of the EOS and the values of the effective coupling constants are most sensitive to the presence of short range correlations, both when we keep and when we omit the contact term in the pion interaction. Finally, we observe that the presence of flexible short range correlations tends to soften the EOS. In fig. 2, the curve "HF-corr-with contact term" appears to contradict this statement. This is because the correlation function used was not flexible enough to respond simultaneously to the repulsive contact interaction and to the attractive component of the interaction. It is also clear that a richer parametrization of the correlation function, such as $f(r)=1-\left(1+\alpha r+\beta r^{2}\right) e^{-\gamma r}$, is required if the contact term is included. Work in this direction are in progress.

It should be said that conclusions drawn from a study of this kind have only qualitative strength since the healing distance constraint imposed on the parameters of the correlation function is not completely unambiguous and since higher order terms of the cluster expansion of the expectation values have not been estimated.

\section{Acknowledgments}

Valuable discussions with S. Moszkowski are gratefully acknowledged. This work was partially supported by FCT (Portugal) under the projects POCTI/FP/FNU/50326/2003, and POCTI/FIS/451/94. PKP is grateful for the friendly atmosphere at Department of Physics, University of Coimbra, where this work was partially done.

\section{APPENDIX}

The angular integrals are given by

$$
\begin{gathered}
A_{i}\left(k, k^{\prime}\right)=B_{i}\left(k, k^{\prime}\right)=2 \pi \frac{g_{i}^{2}}{4 \pi} \int_{0}^{\pi} d \cos \theta \tilde{F}_{i}\left(k, k^{\prime}, \cos \theta\right), \\
C_{i}\left(k, k^{\prime}\right)=2 \pi \frac{g_{i}^{2}}{4 \pi} \int_{0}^{\pi} \cos \theta d \cos \theta \tilde{F}_{i}\left(k, k^{\prime}, \cos \theta\right), \\
I\left(k, k^{\prime}\right)=2 \pi \int_{0}^{\pi} d \cos \theta \tilde{C}_{1}\left(k, k^{\prime}, \cos \theta\right),
\end{gathered}
$$

and

$$
J\left(k, k^{\prime}\right)=2 \pi \int_{0}^{\pi} \cos \theta d \cos \theta \tilde{C}_{1}\left(k, k^{\prime}, \cos \theta\right),
$$


TABLE I: Parameters of nuclear matter. We have used a density dependent parameter $(\mathrm{HF}+\mathrm{corr}) \gamma=600+400 k_{F} / k_{F 0} \mathrm{MeV}$ for the correlation. These parameters were obtained with fixed: $M=939 \mathrm{MeV}, m_{\sigma}=550 \mathrm{MeV}, m_{\omega}=783 \mathrm{MeV}, m_{\rho}=770$ $\mathrm{MeV}, m_{\pi}=138 \mathrm{MeV}, g_{\rho}^{2} / 4 \pi=0.55$ and $f_{\pi}^{2} / 4 \pi=0.08$ at $k_{F 0}=1.3 \mathrm{fm}^{-1}$ with binding energy $E_{B}=\varepsilon / \rho-M=-15.75 \mathrm{MeV}$. The value of $\gamma$ refers to saturation density.

\begin{tabular}{|c|c|c|c|c|c|}
\hline & $g_{\sigma}$ & $g_{\omega}$ & $\alpha$ & $\begin{array}{c}\beta \\
(\mathrm{MeV})\end{array}$ & $\begin{array}{c}\gamma \\
(\mathrm{MeV}) \\
\end{array}$ \\
\hline Hartree & 11.079 & 13.806 & & & \\
\hline $\mathrm{HF}$ & 10.432 & 12.223 & & & \\
\hline $\mathrm{HF}+\operatorname{corr}(\sigma+\omega)$ & 4.4559 & 2.6098 & 13.855 & -2252.448 & 1000 \\
\hline $\mathrm{HF}+\operatorname{corr}(\sigma+\omega+\rho+\pi)$ & 3.1925 & 2.199 & 13.822 & -2258.037 & 1000 \\
\hline $\mathrm{HF}+\operatorname{corr}(\sigma+\omega+\rho+\pi+\pi($ contact term $))$ & 11.662 & 12.930 & 1.5435 & -497.391 & 1000 \\
\hline
\end{tabular}

where

$$
\tilde{F}_{i}\left(\vec{k}, \vec{k}^{\prime}\right)=\int\left[f(r) V_{i}(r) f(r)\right] e^{i\left(\vec{k}-\vec{k}^{\prime}\right) \cdot \vec{r}} d \vec{r} \quad \text { and } \quad \tilde{C}_{1}\left(\vec{k}, \vec{k}^{\prime}\right)=\int(f 2(r)-1) e^{i\left(\vec{k}-\vec{k}^{\prime}\right) \cdot \vec{r}} d \vec{r}
$$

[1] F. Coester, Nucl. Phys. 7421 (1958); F. Coester and H. Kümmel, Nucl. Phys. 17477 (1960).

[2] R. F. Bishop and H. Kümmel, Phys. Today 40(3) 95 (1991); R. F. Bishop, Theor. Chem. Acta 8095 (1991).

[3] F. Villars, "Proceedings of the International School of Physics, 'Enrico Fermi'-Course 23, (1961)." Academic Press, New York, 1963; J.S. Bell, " Lectures on the Many-Body Problem, First Bergen International School of Physics." Benjamin, New York, (1962).

[4] J. da Providencia and C.M. Shakin, Ann. Phys.(NY) 30, 95 (1964).

[5] H. Feldmeier, T. Neff, R. Roth, and J. Schnack, Nucl. Phys. A 632, 61 (1998); T. Neff and H. Feldmeier, Nucl. Phys. 713 311 (2003).

[6] F. Coester, S. Cohen, B.D. Day, and C.M. Vincent, Phys. Rev. C 1, 769 (1970); R. Brockmann and R. Machleidt, Phys. Rev. C 42, 1965 (1990).

[7] R.B. Wiringa, V. Fiks and A. Fabrocini, Phys. Rev. C 38, 1010 (1988); W. Zuo, A. Lejeune, U. Lombardo and J.-F. Mathiot, Nucl. Phys. A 706, 418 (2002).

[8] B.D. Serot, J.D. Walecka, Int. J. Mod. Phys. E6, 515 (1997).

[9] V.G. Neudatchin, I.T. Obukhovsky, V.I. Kukulin and N.F. Golovanova, Phys. Rec. C 11, 128 (1975).

[10] S. A. Moszkowski, Proceedings online of the Conference on Microscopic Approaches to Many-Body Theory (MAMBT), in honor of Ray Bishop, Manchester, UK, http://www.qmbt.org/MAMBT/pdf/Moszkowski.pdf. 2005.

[11] P. K. Panda, J. da Providncia, C. Providncia and D. P. Menezes, Proceedings online of the Conference on Microscopic Approaches to Many-Body Theory (MAMBT), in honor of Ray Bishop, Manchester, UK, http://www.qmbt.org/MAMBT/pdf/Providencia.pdf 2005

[12] P.K. Panda, D.P. Menezes, C. Providência and J. da Providência, Phys. Rev. C 71 015801 (2005); P.K. Panda, D.P. Menezes, C. Providência and J. da Providência, Braz. J. Phys 35873 (2005).

[13] A. Bouyssy, J.-F. Mathiot and N.Van Giai and S. Marcos, Phys. Rev. C 36, 380 (1987).

[14] A. Mishra, P.K. Panda, S. Schramm, J. Reinhardt and W. Greiner, Phys. Rev. C 56, 1380 (1997).

[15] J. da Providencia and C. M. Shakin, Phys. Rev C 4, 1560 (1971); C. M. Shakin, Phys. Rev C 4, 684 (1971).

[16] R. Jastrow, Phys. Rev. 98, 1479 (1955).

[17] S.A. Moszkowski and B.L. Scott, Ann. Phys. (N.Y.), 11, 65 (1960).

[18] H. Bethe, Ann. Rev. Nucl. Sci. 21, 93 (1971).

[19] P. A. M. Guichon, Phys. Lett. B 200, 235 (1988). K. Saito and A.W. Thomas, Phys. Lett. B 327, 9 (1994); P.K. Panda, A. Mishra, J.M. Eisenberg, W. Greiner, Phys. Rev. C 56, 3134 (1997).

[20] S. A. Chin, Ann. Phys. (NY) 108, 301 (1977). 
TABLE II: Ground state properties of nuclear matter at saturation density.

\begin{tabular}{|c|c|c|c|c|c|c|}
\hline & $M^{*} / M$ & $\begin{array}{c}\mathcal{T} / \rho_{B}-M \\
(\mathrm{MeV})\end{array}$ & $\begin{array}{l}\mathcal{V}_{d} / \rho_{B} \\
(\mathrm{MeV})\end{array}$ & $\begin{array}{l}\mathcal{V}_{e} / \rho_{B} \\
(\mathrm{MeV})\end{array}$ & $\begin{array}{l}\mathcal{T}^{C} / \rho_{B} \\
(\mathrm{MeV})\end{array}$ & $\begin{array}{c}\text { contact term } \\
(\mathrm{MeV})\end{array}$ \\
\hline Hartree & 0.540 & 8.11 & -23.86 & & & \\
\hline $\mathrm{HF}$ & 0.515 & 5.87 & -37.45 & 15.83 & & \\
\hline $\mathrm{HF}+\operatorname{corr}(\sigma+\omega)$ & 0.625 & 15.95 & -73.12 & 20.46 & 19.96 & \\
\hline $\mathrm{HF}+\operatorname{corr}(\sigma+\omega+\rho+\pi)$ & 0.645 & 16.41 & -17.76 & -34.57 & 20.16 & \\
\hline $\mathrm{HF}+\operatorname{corr}(\sigma+\omega+\rho+\pi+\pi($ contact term $))$ & 0.517 & 6.67 & -42.30 & 11.11 & 2.53 & 6.21 \\
\hline
\end{tabular}

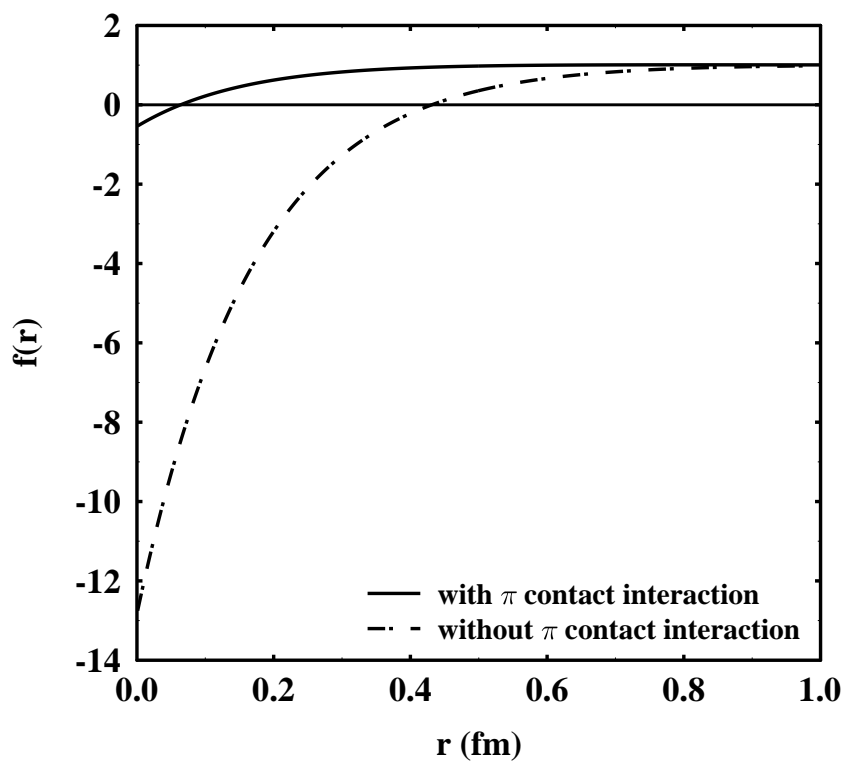

FIG. 1: The correlation function $f(r)$ for the calculation with and without the delta term of the $\pi$ contribution. 


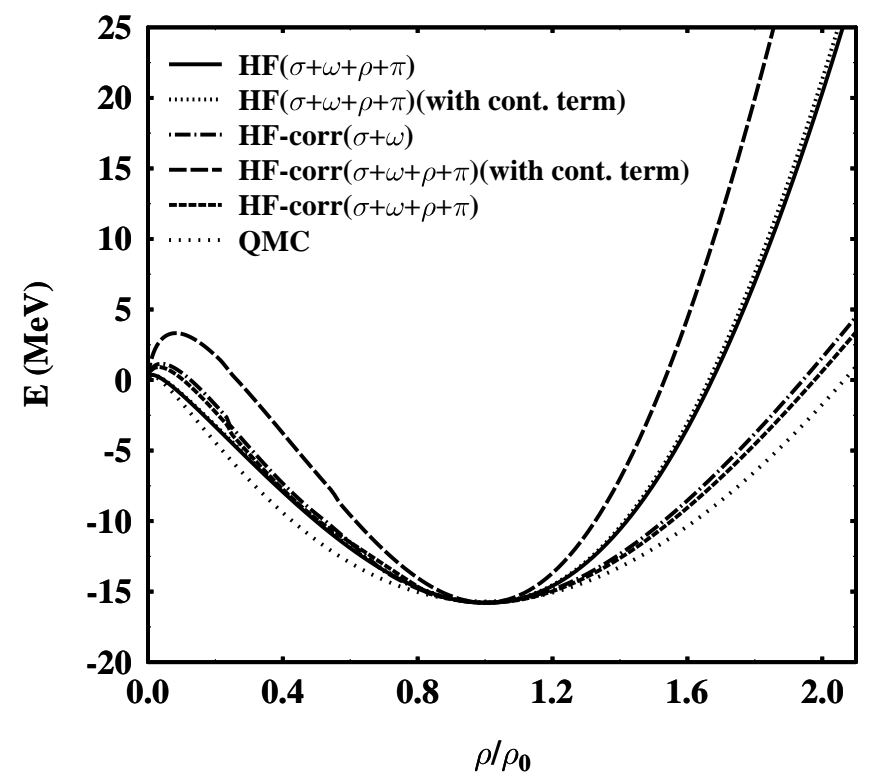

FIG. 2: The equation of state with and without short range correlations.

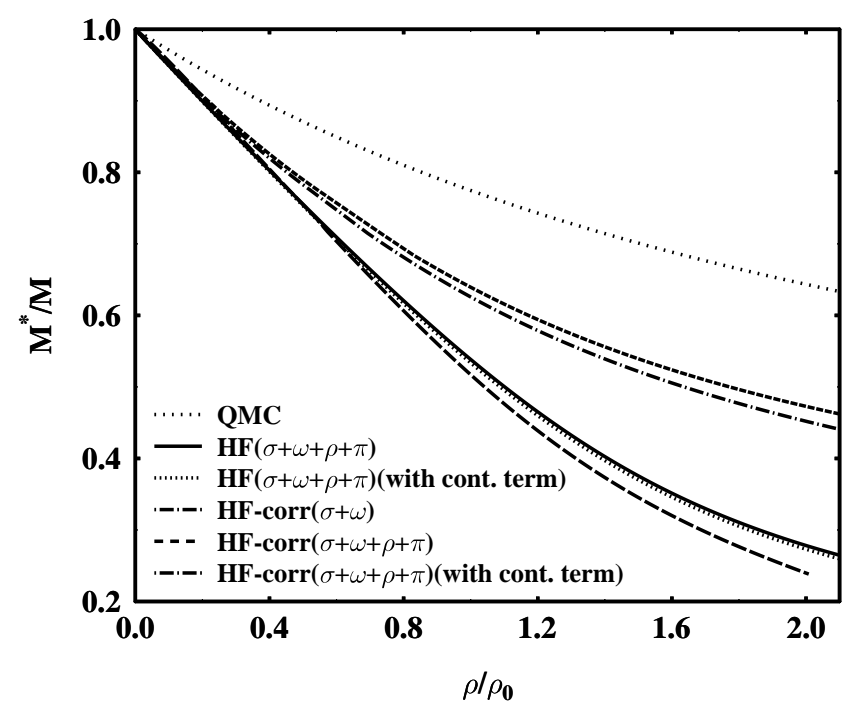

FIG. 3: Effective mass as a function of density. 


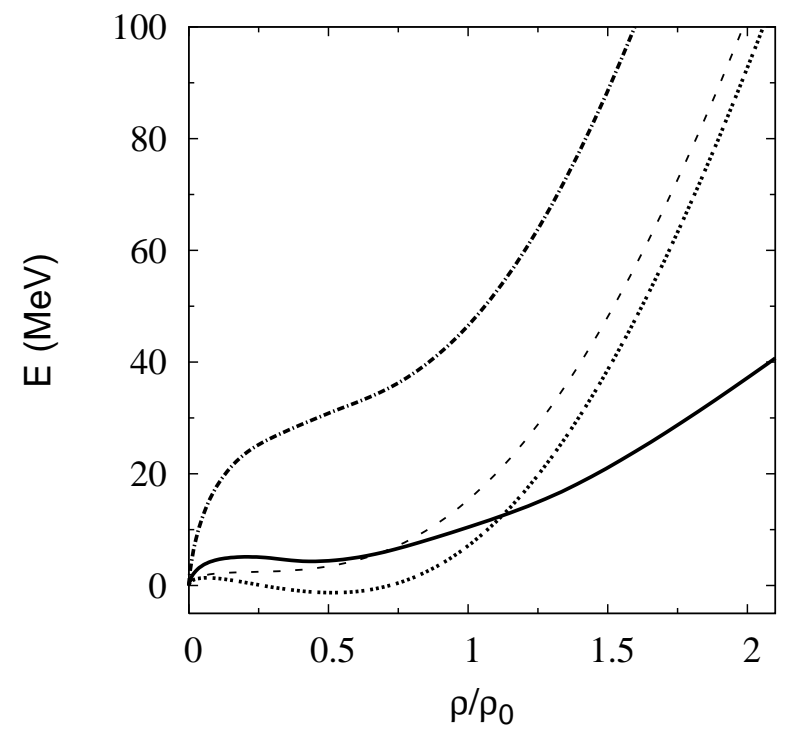

FIG. 4: Equation of state of neutron matter (solid line: $\sigma+\omega+\rho+\pi+$ correlation (without delta), dash-dotted line: $\sigma+\omega+$ $\rho+\pi+$ correlation (with delta), dotted line: $\sigma+\omega+\rho+\pi$ (without delta), dashed line: $\sigma+\omega+\rho+\pi$ (with delta) 